\title{
New insights into the role of Portlandite in the cement system: elastic anisotropy, thermal stability, and structural compatibility with C-S-H
}

Qi Zheng ${ }^{1}$, Jinyang Jiang ${ }^{1 *}$, Guangyuan $\mathrm{Xu}^{1}$, Jin $\mathrm{Yu}^{1}$, Luping Tang ${ }^{3}$, Shaofan $\mathrm{Li}^{2 *}$

${ }^{1}$ School of Materials Science and Engineering, Southeast University, Nanjing 211189,

P.R. China

${ }^{2}$ Department of Civil and Environmental Engineering, University of California at Berkeley, Berkeley, CA 94720-1710, USA

${ }^{3}$ Department of Architecture and Civil Engineering, Chalmers University of Technology, Sweden 


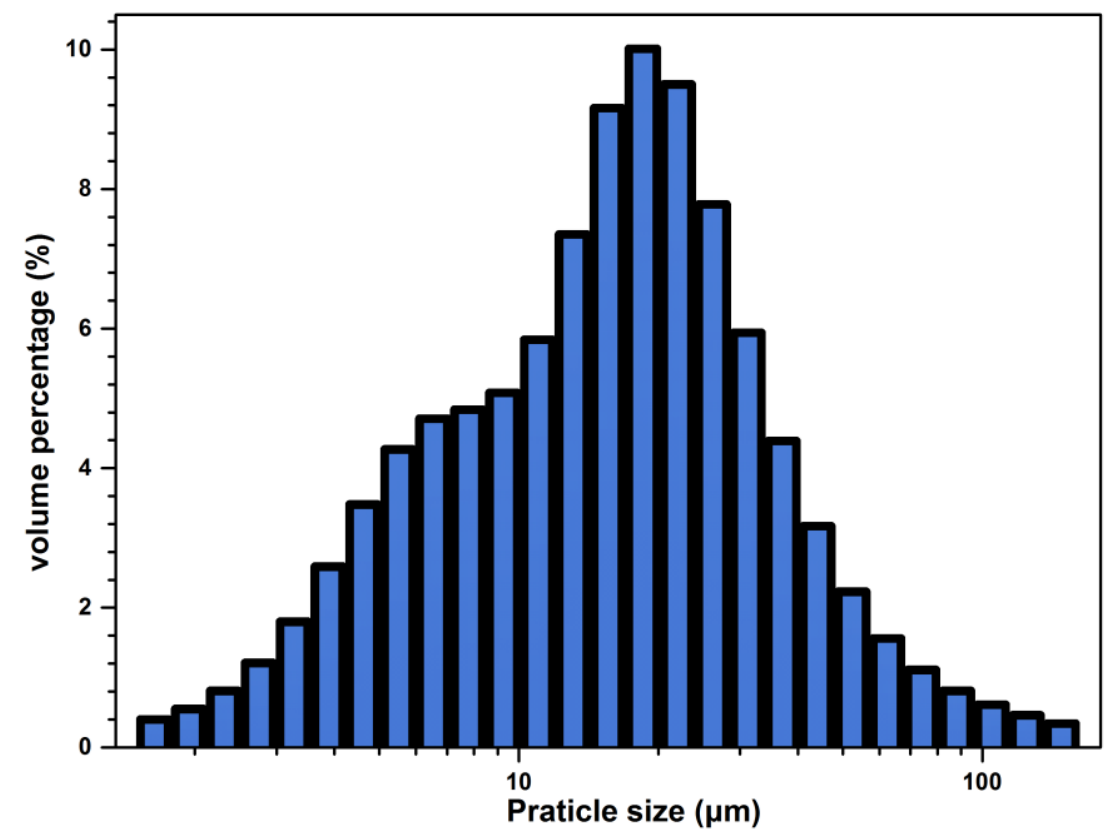

Figure S1. Particle size distribution of $\mathrm{C}_{3} \mathrm{~S}$.

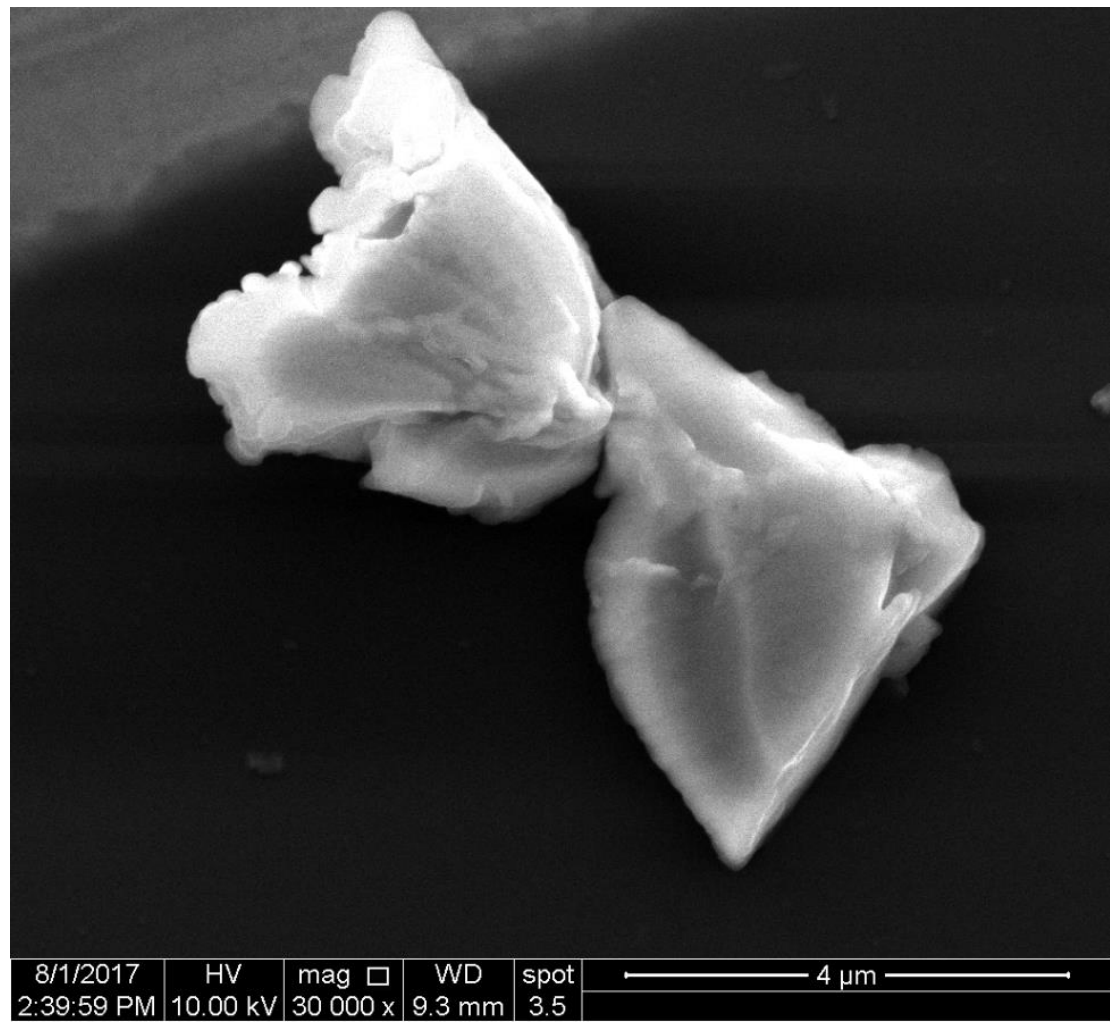

Figure S2. SEM image of $\mathrm{C}_{3} \mathrm{~S}$ grains. These particles are in random shape with various edges and corners. 


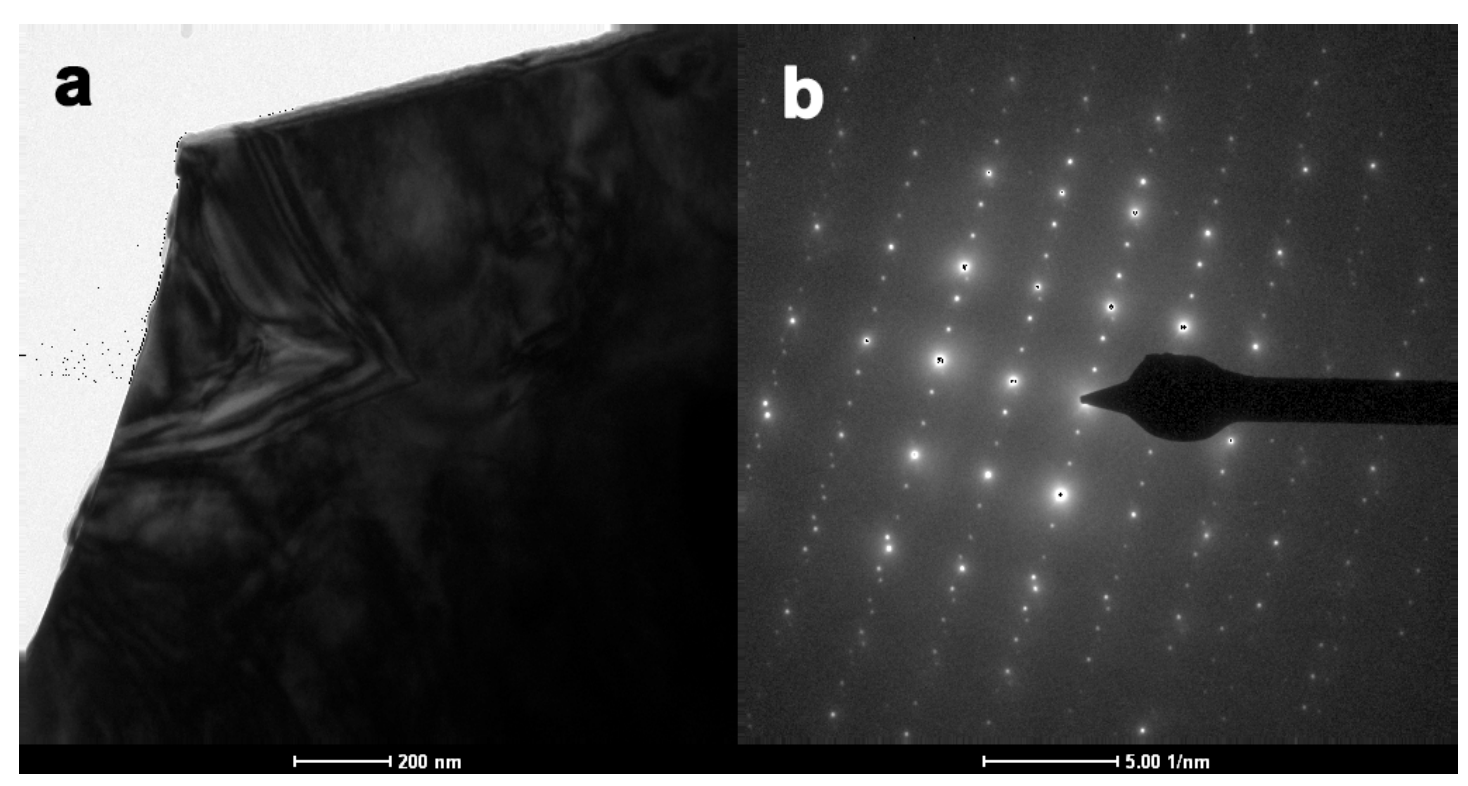

Figure S3. TEM images of $\mathrm{C}_{3} \mathrm{~S}$. (a) Bright field image of $\mathrm{C}_{3} \mathrm{~S}$. Since the thickness of $\mathrm{C}_{3} \mathrm{~S}$ is quite big, the electron beam cannot transmit through, resulting in black background. (b) Selected area of electron diffraction pattern of $\mathrm{C}_{3} \mathrm{~S}$ at edge. The pattern indicates that $\mathrm{C}_{3} \mathrm{~S}$ is in a crystalline state.

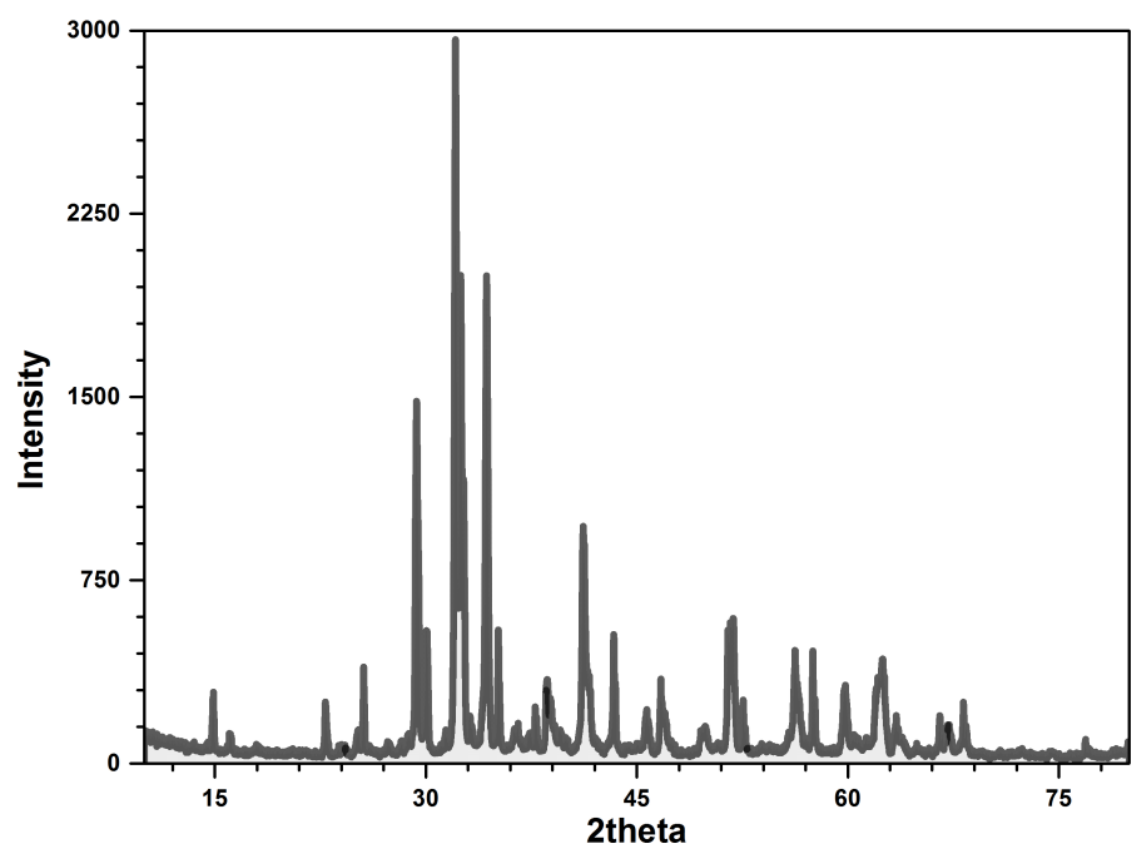

Figure $\mathrm{S} 4$ : XRD pattern of $\mathrm{C}_{3} \mathrm{~S}$, indicating it is a perfect crystal with featured peaks. 

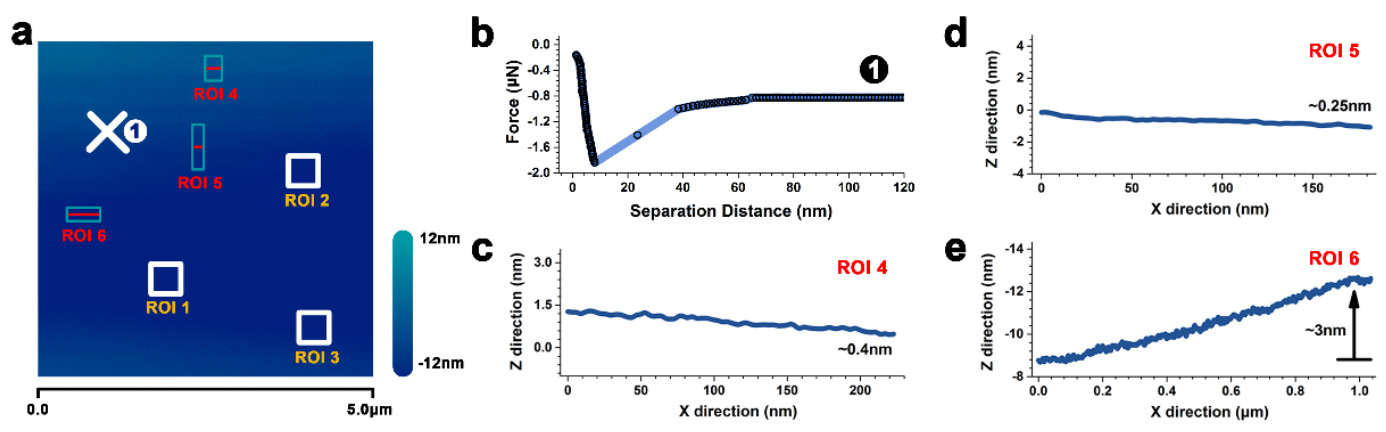

Figure S5: AFM image of mica substrate. (a) Top view of mica: Seven ROIs are randomly selected and marked in cross and squares. (b) Force-deflection curve of area 1 , the indentation modulus is determined by the scope, to be 26.426 GPa ,ranged from $0 \mathrm{~nm}$ to $12 \mathrm{~nm}$. (c-e) The smoothness is checked in different ROIs. Some regions like ROI1-3 are likely chosen for dropcasting portlandite crystals.

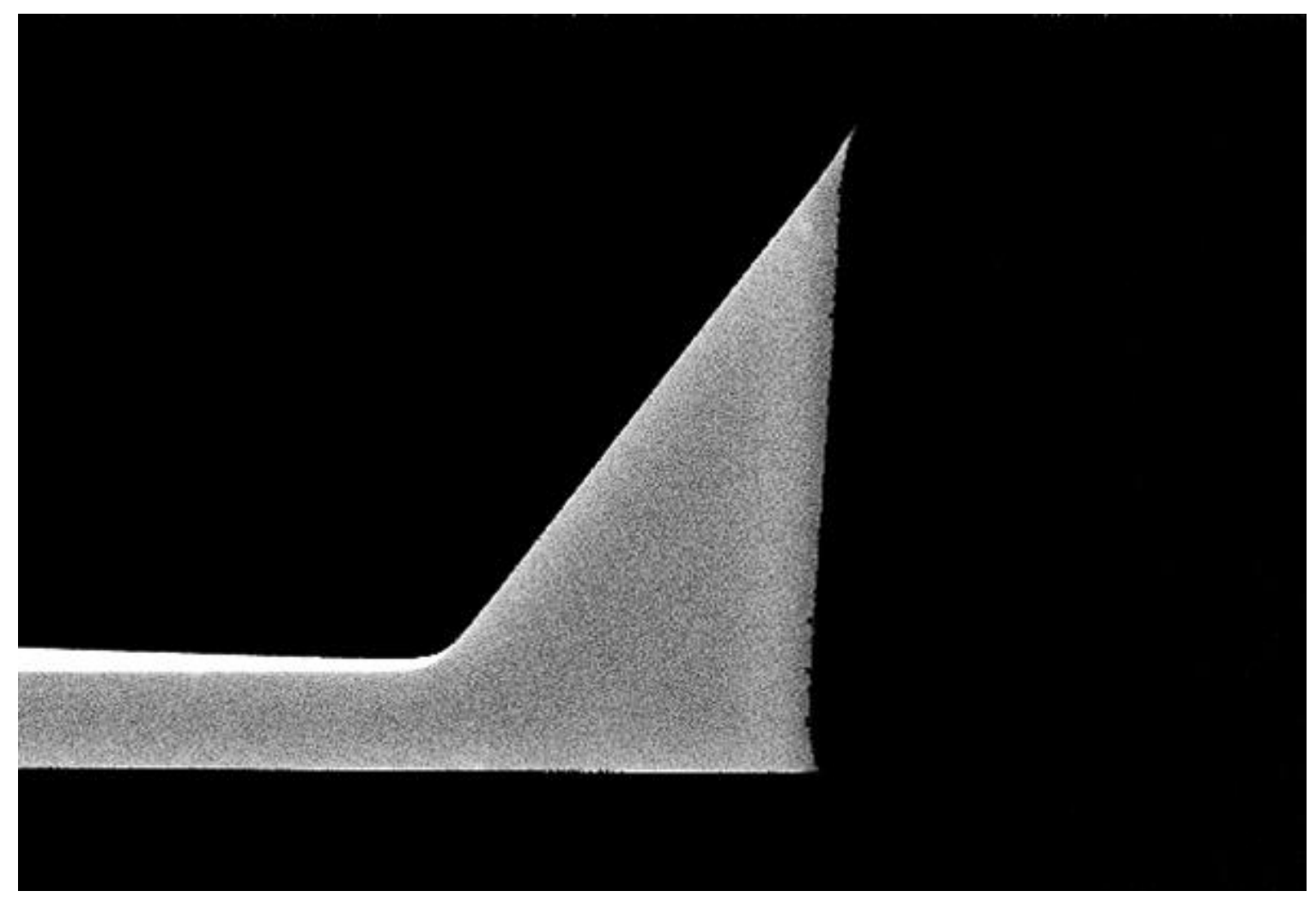

Figure S6. SEM image of the tip of AFM platform. More details can be found on https://www.brukerafmprobes.com. 


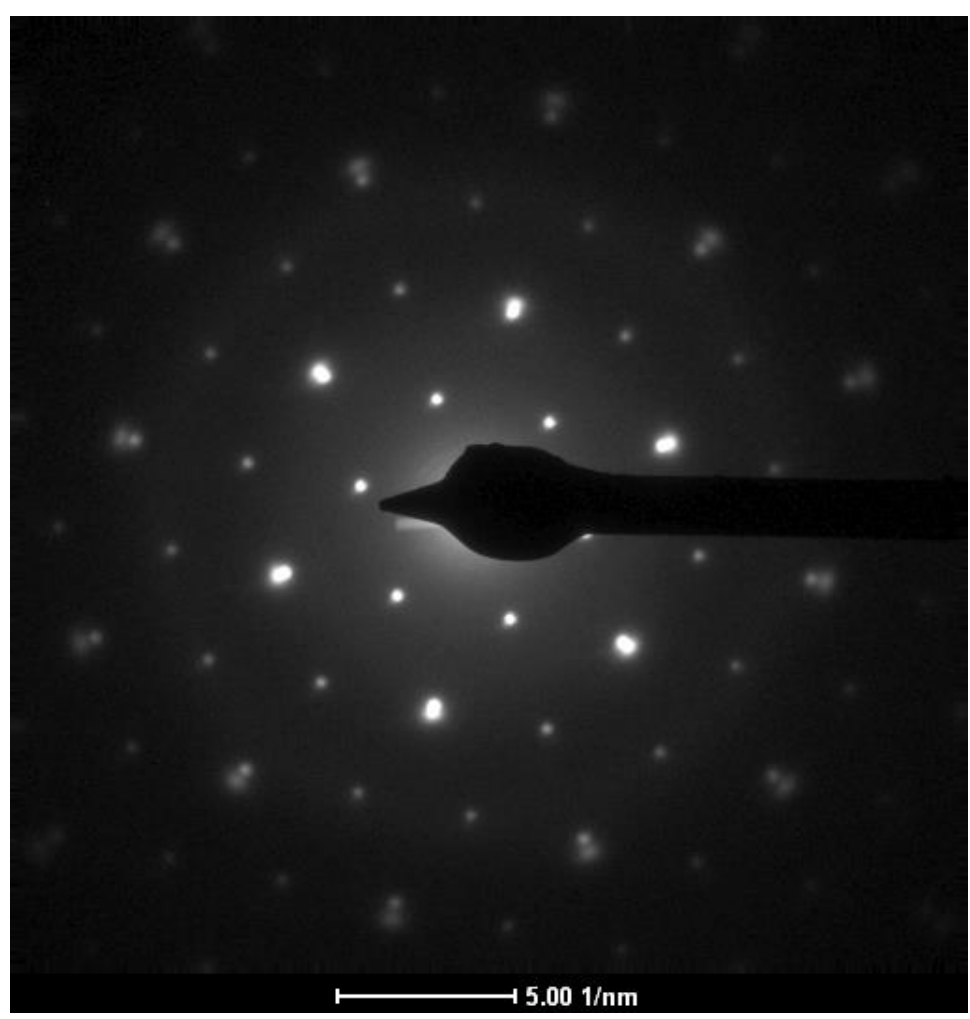

Figure S7. Selected area diffraction pattern of portlandite crystal, exhibiting good crystalline degree.
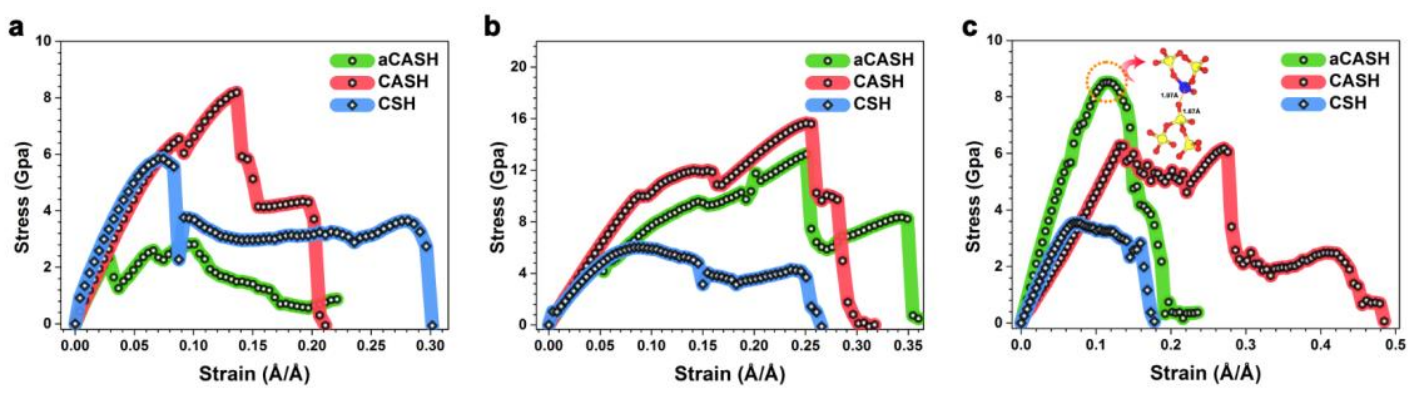

Figure S8. Uniaxial tensile behavior of C-S-H gel in different directions. (a) in a direction, (b) in b direction and (c) in $\mathbf{c}$ direction. C-S-H is an anisotropic material that it is much ductile in $\mathbf{a}, \mathbf{b}$ directions while it exhibits brittle fracture characters in $\mathbf{c}$ direction. To be specific, the yield stress and yield strain are determined by the first peak before the plateau and the failure point is chosen by the sudden break or total failure in the tension. 